\title{
Nested calcium dynamics support daily cell unity and diversity in the suprachiasmatic nuclei of free-behaving mice
}

Lama El Cheikh Hussein ${ }^{1}$, Pierre Fontanaud ${ }^{1,2}$, Patrice Mollard ${ }^{1,2}$ and Xavier Bonnefont ${ }^{1 *}$

${ }^{1}$ Institut de Génomique Fonctionnelle, Université de Montpellier, CNRS, INSERM, Montpellier, France

${ }^{2}$ BioCampus Montpellier, Université de Montpellier, CNRS, INSERM, Montpellier, France

*e-mail: Xavier.Bonnefont@igf.cnrs.fr

The suprachiasmatic nuclei (SCN) of the anterior hypothalamus host the circadian pacemaker that synchronizes mammalian rhythms with the day-night cycle. SCN neurons are intrinsically rhythmic, thanks to a conserved cell-autonomous clock mechanism. In addition, circuit-level emergent properties confer a unique degree of precision and robustness to SCN neuronal rhythmicity. However, the multicellular functional organization of the SCN is not yet fully understood. Although SCN neurons are well coordinated, experimental evidences indicate that some neurons oscillate out of phase in SCN explants, and possibly to a larger extent in vivo. Here, we used microendoscopic $\mathrm{Ca}^{2+}{ }_{\mathrm{i}}$ imaging to investigate SCN rhythmicity at a single cell resolution in free-behaving mice. We found that SCN neurons in vivo exhibited fast $\mathrm{Ca}^{2+}{ }_{\mathrm{i}}$ spikes superimposed upon slow changes in baseline $\mathrm{Ca}^{2+}{ }_{\mathrm{i}}$ levels. Both spikes and baseline followed a time-of-day modulation in many neurons, but independently from each other. Daily rhythms in basal $\mathrm{Ca}^{2+}{ }_{i}$ were well coordinated, while spike activity from the same neurons peaked at multiple times of the light cycle, and unveiled clock-independent interactions at the multicellular level. Hence, fast $\mathrm{Ca}^{2+}{ }_{i}$ spikes and slow changes in baseline $\mathrm{Ca}^{2+}{ }_{i}$ levels highlighted how diverse activity patterns could articulate within the temporal network unity of the SCN in vivo, and provided support for a multiplex neuronal code in the circadian pacemaker. 


\section{Introduction}

Circadian clocks rely on a conserved molecular mechanism expressed in virtually all body cells (1). In the suprachiasmatic nuclei (SCN) of the anterior hypothalamus, circuit-level interactions confer a unique degree of precision and robustness to circadian neuronal rhythms, which turns cellautonomous oscillators into the central pacemaker that synchronizes mammalian physiology and behaviors with the day-night cycle (2-6). Deciphering the multicellular functioning of the SCN will thus constitute a milestone on the way from clock genes to complex, overt rhythms.

SCN neurons are coordinated but they are not always perfectly in phase. Stereotyped circadian waves in clock gene expression, intracellular calcium concentration $\left(\mathrm{Ca}^{2+}{ }_{i}\right)$, and spontaneous firing rate of action potentials outline an approximate 6-hour phase gradient throughout SCN slices (3, 7-10). However, recent evidences challenged the rhythmic unity in the $\mathrm{SCN}$, with some neurons exhibiting extreme phase lag. Phaseoids were defined as groups of neurons expressing the circadian clock protein PERIOD2 stably out of phase with their neighbors (11). Similarly, some SCN neurons appear electrophysiologically more active during nighttime, in total opposition of phase relative to the ensemble rhythm $(12,13)$. Last but not least, the circadian amplitude in multi-unit electrophysiological activity is dramatically downsized in vivo as compared to in vitro preparations (14), suggesting reduced neuronal synchronicity in living animals. How such a diversity in neuronal activity in vivo articulates within a coherent SCN network remains unclear.

Typically, fast $\mathrm{Ca}^{2+}{ }_{i}$ spikes driven by action potentials provide a fairly reliable estimate of neuronal activity. While circadian variations in $\mathrm{Ca}^{2+}{ }_{i}$ were extensively described in individual SCN neurons ex vivo $(7,8,15-17)$, and at a neuronal population level in vivo $(18,19)$, the spatiotemporal organization of $\mathrm{SCN} \mathrm{Ca}{ }^{2+}{ }_{i}$ spikes has been barely investigated, possibly because of technical issues with BAPTA-based $\mathrm{Ca}^{2+}{ }_{\mathrm{i}}$ probes $(20,21)$. Here we performed microendoscopic imaging of the $\mathrm{Ca}^{2+}{ }_{\mathrm{i}}$ indicator GCamp6f at a single-cell resolution through a graded-index (GRIN) lens aimed at the SCN of freely behaving mice. Fast $\mathrm{Ca}^{2+}{ }_{i}$ spikes revealed unexpected diversity in daily neuronal activity patterns, and circuit-level signaling all around the light-dark cycle. Yet, we found that this diversity builds upon a highly coordinated wave in baseline $\mathrm{Ca}^{2+}{ }_{i}$ levels in the same cells. Together, $\mathrm{Ca}^{2+}{ }_{i}$ spikes and baseline $\mathrm{Ca}^{2+}{ }_{\mathrm{i}}$ levels were independently modulated with the time of day in vivo, and reconciled the apparent discrepancy between functional cell diversity and unity in the SCN cell network.

\section{Results}

After surgical preparation (see methods), mice were trained to the imaging setup for at least 3 weeks. They exhibited low levels of circulating corticosterone during their resting phase, which increased at the beginning of their active phase (Fig. S1). Moreover, longitudinal monitoring of the global GCamp6f signal from the SCN ( $n>10$ mice) revealed daily changes, with peak and nadir in the 
middle of the light and dark phase (Fig. 1a,b), respectively, resembling the typical $\mathrm{Ca}^{2+}{ }_{i}$ rhythm recorded at the neuron population level using in vivo fiber photometry $(18,19)$. Hence, GRIN lensimplanted mice were free of chronic stress and maintained normal daily physiology under our experimental conditions.

All the recorded SCN neurons displayed short-lived spikes in GCamp6f (Movie 1), superimposed upon slow-evolving changes in basal fluorescence (Fig. 1c). Remarkably, some neurons were more active during daytime and others during nighttime, even in the same field of view (Fig. 1C). To assess the temporal patterns in $\mathrm{Ca}^{2+}{ }_{\mathrm{i}}$ activity, we estimated the frequency of GCamp6f spikes from 155 individual SCN neurons from four mice, recorded longitudinally over at least 24 hours, for 1 minute every 30 minutes. Spikes could occur at all times of day, with $38-69 \%$ of the neurons exhibiting a significant daily organization in spike frequency (Fig. 1d-f, Fig. S2-5), as assessed by non-parametric JTK-Cycle analysis (22). The large dispersion of peak activity phases from these neurons ( $p>0.1$ Moore's modified Rayleigh test, Fig. 1e,f) revealed unforeseen diversity of daily patterns in SCN neuronal activity, and underscored the relevance in vivo of night-active neuron populations previously reported in slices $(12,13)$.

Importantly, the daily organization in basal $\mathrm{Ca}^{2+}{ }_{i}$ in the same $\mathrm{SCN}$ neurons differed markedly from that in fast spiking activity. Up to 145 out of the 155 recorded neurons (70\% to 100\%) displayed a significant daily pattern in baseline GCamp6f levels that always reached a maximum during daytime (Fig. 1g-i, Fig. S2-5), in phase with the global GCamp6f signal. First, the overall phase difference between all four SCN ( $p<10-7, F(3,141)$, Watson-Williams F-test) suggested subtle inter-individual variability, or topographical heterogeneity that was reminiscent of the phase gradient in circadian $\mathrm{Ca}^{2+}{ }_{i}$ levels observed in SCN slices $(7,8)$. However, the narrow phase distribution of maximum baseline GCamp6f levels, centered around zeitgeber time ZT=6 (with ZT=0 at lights on 95\% confidence interval [ZT=03:28, ZT=07:11], $\mathrm{p}<0.001$ Moore's modified Rayleigh test), underscored the remarkable unity of daily changes in baseline $\mathrm{Ca}^{2+}{ }_{i}$ levels in individual $\mathrm{SCN}$ neurons. This resulted in a stratified phase-lag relationship with the daily variation in spike frequency (Fig. $1 \mathrm{~h}$ ), indicating that fast $\mathrm{Ca}^{2+}{ }_{i}$ spike activity and basal $\mathrm{Ca}^{2+}{ }_{i}$ levels in $\mathrm{SCN}$ neurons were modulated in vivo with the time of day, independently from each other.

To gain further insight into the contribution of spikes to the daily $\mathrm{Ca}^{2+}{ }_{i}$ rhythm in $\mathrm{SCN}$ neurons, relatively to baseline changes, we measured the amplitude of changes in GCamp6f fluorescence in each one-minute activity recording (Fig. 2a). This parameter followed a daily organization in about half (33-52\%) of the recorded neurons (Fig. 2b-d, Fig. S2-5), correlated with spike frequency (Spearman $r=0.94, p<0.0001$ ) (Fig. 2e). Spiking activity was generally one-to-two orders of magnitude smaller than the variation in basal GCamp6f fluorescence measured over 24 hours in the same neuron (Fig. 2f). Therefore, spikes contributed moderately to the daily rhythmicity in fluorescence, regardless of 
whether they occurred at high or low baseline levels (Fig. 2g,h). These data indicated that slow changes in basal $\mathrm{Ca}^{2+}{ }_{\mathrm{i}}$ levels and fast $\mathrm{Ca}^{2+}{ }_{i}$ spikes were nested dynamics in $\mathrm{SCN}$ neurons, with spikes providing cellular diversity to the coherent multicellular rhythm in baseline. Together, these $\mathrm{Ca}^{2+}{ }_{i}$ dynamics supported daily functional unity and diversity in the SCN cell network in vivo.

Next, we explored the spatiotemporal organization of GCampf spikes at the multicellular scale to question the circuit-level significance of the fast $\mathrm{Ca}^{2+}{ }_{i}$ activity in the SCN. We found that fast GCamp6f spikes could occur within the same time frame in clusters of SCN neurons that partially overlapped each other (Fig. 3a, Movie 1). This co-activity corresponded to noticeable changes in the global field signal (Fig. 3b), indicating that many neurons were recruited in and out of focus at the same time. The extent of co-activity clusters in the field of view, as expressed in cell.clusters/cell/min, correlated positively to the density of neuronal activity (Spearman $r=0.71, p<0.0001$, Fig. 3c), and spread evenly at all times of the light-dark cycle ( $p>0.5$, Moore's modified Rayleigh test, Fig. $3 d$ ). Since this quantitative assessment involved only co-activity detected above chance threshold (see methods), the correlation between co-activity and activity density resulted more likely from deterministic mechanisms, rather than random $\mathrm{Ca}^{2+}{ }_{i}$ spike coincidence in highly active neurons. These data suggested that circuit-level signaling involving $\mathrm{Ca}^{2+}{ }^{2}$-dependent transduction pathways synchronized SCN neurons all around the clock.

To address the mechanisms underlying the concerted activation of SCN neurons, we interrogated the multicellular organization of $\mathrm{Ca}^{2+}{ }_{i}$ spikes under the conditions of an altered circadian clock. First, clusters of co-activity persisted during jetlag, when the global GCamp6f rhythm was progressively resetting to a new light phase (Fig. S6). Second, we conducted an independent set of GCamp6f measurements in the SCN of globally clockless Cry1-/- Cry2-/- mutant mice ( $n=4$ mice). As in control mice, we observed large groups of co-active neurons that produced global events in the whole field signal (Fig. 3e). Hence, circadian clock-independent $\mathrm{Ca}^{2+}{ }_{i}$ signaling promoted extensive cell-cell coordination in the $\mathrm{SCN}$ of free-behaving mice.

\section{Discussion}

Individuating part-whole relations in neural circuits has become an important challenge (23, 24). Our results uncovered an unprecedented relationship between fast and slow $\mathrm{Ca}^{2+}{ }_{i}$ dynamics that supported diversity in neuronal activity within a temporal network unity, in the SCN of free-behaving mice (Fig. 4).

At the single cell level, fast $\mathrm{Ca}^{2+}{ }_{i}$ spikes underscored the wide phase distribution in daily activity profiles, with a surprisingly high proportion of night-active neurons in our conditions, which somehow corroborated the elevated action potential firing rate from the SCN of live animals during nighttime (14). At the multicellular level, fast $\mathrm{Ca}^{2+}{ }_{i}$ spikes provided evidence of synchronization that might 
contribute to the unity of daily rhythms in baseline $\mathrm{Ca}^{2+}{ }_{i}$. Synchronized $\mathrm{Ca}^{2+}{ }_{i}$ spikes appeared at all times of day, and persisted under circadian clock disruption, in support of clock-independent circuit-level properties of the circadian pacemaker $(10,25)$. These results highlighted the relevance of $\mathrm{Ca}^{2+}{ }_{i}$ signaling across multiple timescales in the SCN.

The differential modulation of $\mathrm{Ca}^{2+}{ }_{i}$ spikes and basal $\mathrm{Ca}^{2+}{ }_{i}$ with the time of day suggested their relying on different mechanisms. Fast $\mathrm{Ca}^{2+}{ }_{i}$ spikes in $\mathrm{SCN}$ neurons were typically associated with electrophysiological firing, though in a non-linear relationship as individual spikes occurring at high frequency happen to sum up in amplitude (21). The correlation observed here between $\mathrm{Ca}^{2+}{ }_{i}$ spike frequency and signal amplitude was consistent with this assumption. Conversely, circadian $\mathrm{Ca}^{2+}{ }_{i}$ rhythmicity, which we found was chiefly shaped by variations in basal $\mathrm{Ca}^{2+}{ }_{i}$, was reportedly dependent on intracellular stores and resistant to blockers of voltage-dependent channels $(16,17)$. Hence, action potential-driven fast $\mathrm{Ca}^{2+}{ }_{i}$ spikes of low amplitude might built upon large and slow waves in daily release from internal $\mathrm{Ca}^{2+}{ }_{i}$ stores, although the source of each $\mathrm{Ca}^{2+}{ }_{i}$ dynamics likely differs among the various $\operatorname{SCN}$ neuronal subtypes $(8,26,27)$.

$\mathrm{Ca}^{2+}{ }_{\mathrm{i}}$ is a versatile second messenger that encodes a large array of cell functions $(23,28)$. Here, fast $\mathrm{Ca}^{2+}{ }_{i}$ spikes offered a tremendous extension of the repertoire of $\mathrm{Ca}^{2+}{ }_{i}$ signaling in the $\mathrm{SCN}$, from the typical binary alternation of up and down states, toward the possibility of an activity-dependent multiplex code (Fig. 4), deciphered by the composite tuning of downstream molecular effectors that have already been reported in SCN neurons $(29,30)$.

Since the SCN are composed of multiple neuronal subtypes, based on gene expression profiles or neuropeptide content (31-35), the question arises as to whether neurons exhibiting the same $\mathrm{Ca}^{2+}{ }_{i}$ code belong to the same subtype and/or eventually constitute functional units gating specific body rhythms (13, 36-39). In a parallel study, Stowie et al. investigated circadian $\mathrm{Ca}^{2+}{ }_{\mathrm{i}}$ rhythmicity in arginine vasopressin-expressing SCN neurons in vivo (40). Further experiments implementing this cell typespecific approach will be necessary to monitor and manipulate $\mathrm{Ca}^{2+}{ }_{i}$ signaling in identified $\mathrm{SCN}$ neurons coupled to specific circadian outputs.

\section{Materials and Methods}

Animals

All mouse experiments complied with the European Directive 2010/63/UE, and were registered under the reference APAFIS\#15032-2018050918181227 v2. Male C57BI/6J mice (5-6 weeks old) were purchased from Janvier Labs (Le Genest-Saint-Isle, France). Cry1-/-Cry2-/- mice (41) were produced by breeding double-heterozygous males and females from our colony maintained in a C57B/6/J background for more than 20 generations (42). All mice were housed in ventilated micro-isolator cages, under a $12 \mathrm{~h}: 12 \mathrm{~h}$ light:dark cycle, with free access to food and water. 


\section{Surgery}

At the age of 8 weeks, mice were anesthetized with a cocktail of Ketamine (Imalgene $500,75 \mathrm{mg} / \mathrm{kg}$ ) and Xylazine (Rompun, $10 \mathrm{mg} / \mathrm{kg}$ ). A skin incision exposed the skull, and small craniotomies were made dorsal to the injection site with a drill mounted on a stereotaxic apparatus. The virus solution (1000 nl, titer $\geq 1013 \mathrm{vg} / \mathrm{ml}$, \#100836-AAV9 from Addgene) was injected in the SCN (ML+/-0.2, AP 0, DV -5.7) at a rate of $50 \mathrm{nl} /$ minute using a microinjector-controlled syringe (micro-4, World Precision Instruments) and needle (Nanofil 33G beveled needle, World Precision Instruments). The needle was kept in place for 10 minutes following injection to allow suitable diffusion, and reduce backflow during withdraw. Mice were then implanted with a graded-index (GRIN) lens (diameter $0.6 \mathrm{~mm}$, length $7.44 \mathrm{~mm}$, working distance $150 \mu \mathrm{m}$, \#AB000436 from GRINTECH), aimed 50-100 $\mu \mathrm{m}$ dorsally to the virus injection site. The lens was stabilized with dental cement (Metabond) and Kwik-Silsealant (World Precision Instruments). After 3-4 weeks, mice were anesthetized for placement of the microendoscope baseplate (\#1050-004638, Inscopix), and a baseplate cover (\#1050-004639, Inscopix) was used to protect the lens when not in use.

After surgery, mice were housed in individual cages, and regularly habituated to the imaging setup, with a dummy microendoscope (DMS-2, Inscopix) for at least 3 weeks. To ascertain well-being and normal daily physiology under these conditions, tail-tip blood ( $6 \mu \mathrm{l})$ was collected from 4 mice at ZT7 and ZT12 to check corticosterone levels (ELISA kit From Assaypro).

\section{Microendoscopic data acquisition and processing}

Images were acquired with a head-mounted miniaturized microscope (nVista, Inscopix) at 4 frames per second over one minute (240 ms exposure time, 10-20\% LED illumination, 1.5-2.5x gain). Oneminute movies were spatially filtered (high-pass filter, cutoff at $40 \mu \mathrm{m}$ ), corrected for motion artifacts (Inscopix Data Processing Software), and saved as stacks of frames in TIFF format. Regions of interest (ROI) were defined and applied to every stack (ImageJ), and numerical data were saved as text files for further processing in Matlab (MathWorks).

\section{Data analysis and statistics}

A Butterworth low-pass filter was applied (cutoff frequency at $1.1 \mathrm{~Hz}$ ). After detrending, the baseline level for each one-minute recording was defined as the 5th percentile value, and the signal amplitude as the difference between the 5th and 95th values. Fast spikes were identified with a two-threshold procedure as fast (threshold 1, above the 90th percentile of all derivative values from all ROls over the course of a 24-hour period) and large events (threshold 2: peak value above 2 standard deviations of 
all the raw signals from the considered ROI). The onset of spikes (derivative crossing above 0) was computed as their time of occurrence.

$\mathrm{Ca}^{2+}{ }_{i}$ spikes occurring in a high-activity frame in a statistically significant number of neurons delineated the clusters of coactivity. The threshold corresponding to a significance level of $p<0.05$ was defined as the number of activated cells in a single frame that exceeded only $5 \%$ of 500 surrogate datasets obtained by randomly transposing intervals of activity within each cell (43). The extent of clusters of coactivity was computed in Cell.Clusters/Cell/min, as the sum of cells participating in any of the coactive events in a one-minute recording, normalized by the total number of cells recorded in the field.

Non-parametric JTK-Cycle analysis (22) was used to estimate the daily phase of the GCamp6f signal parameters from datasets recorded over one 24-hour period. Circular analysis and statistics were conducted using Oriana (Kovach Computing Services). Other numerical and statistical analyses were conducted with IgorPro (WaveMetrics, Inc), Prism 7 (GraphPad Software, Inc).

\section{Acknowledgements}

This work was supported by Centre National de la Recherche Scientifique (P.M., P.F., and X.B.), Agence Nationale pour la Recherche (ANR) grant nos. ANR-15-CE14-0012 and ANR-18-CE14-0017-01, and France Biolmaging ANR-10-INSB-04 "Investments for the Future" to P.M., and a fellowship from Secours Populaire Libanais to L.E.C.H. We thank Ombeline Hoa and Pauline Campos for advices with GRIN lens implantation, and the iExplore and IPAM platforms in Montpellier for technical support. We are grateful to Robert J. Lucas for reading an earlier draft of the manuscript. Alec J. Davidson is acknowledged for sharing data prior to publication.

\section{References}

1. Takahashi JS (2017) Transcriptional architecture of the mammalian circadian clock. Nat Rev Genet 18(3):164-179.

2. El Cheikh Hussein L, Mollard P, \& Bonnefont X (2019) Molecular and Cellular Networks in The Suprachiasmatic Nuclei. Int J Mol Sci 20(8).

3. Hastings MH, Maywood ES, \& Brancaccio M (2018) Generation of circadian rhythms in the suprachiasmatic nucleus. Nat Rev Neurosci 19(8):453-469.

4. Herzog ED, Aton SJ, Numano R, Sakaki Y, \& Tei H (2004) Temporal precision in the mammalian circadian system: a reliable clock from less reliable neurons. J Biol Rhythms 19(1):35-46.

5. Honma S, Nakamura W, Shirakawa T, \& Honma K (2004) Diversity in the circadian periods of single neurons of the rat suprachiasmatic nucleus depends on nuclear structure and intrinsic period. Neurosci Lett 358(3):173-176.

6. Liu AC, et al. (2007) Intercellular coupling confers robustness against mutations in the SCN circadian clock network. Cell 129(3):605-616.

7. Brancaccio M, Maywood ES, Chesham JE, Loudon AS, \& Hastings MH (2013) A Gq-Ca2+ axis controls circuit-level encoding of circadian time in the suprachiasmatic nucleus. Neuron 78(4):714-728. 
8. Enoki R, et al. (2017) Synchronous circadian voltage rhythms with asynchronous calcium rhythms in the suprachiasmatic nucleus. Proc Natl Acad Sci U S A 114(12):E2476-E2485.

9. Evans JA, Leise TL, Castanon-Cervantes O, \& Davidson AJ (2013) Dynamic interactions mediated by nonredundant signaling mechanisms couple circadian clock neurons. Neuron 80(4):973-983.

10. Pauls S, et al. (2014) Differential contributions of intra-cellular and inter-cellular mechanisms to the spatial and temporal architecture of the suprachiasmatic nucleus circadian circuitry in wild-type, cryptochrome-null and vasoactive intestinal peptide receptor 2-null mutant mice. Eur J Neurosci 40(3):2528-2540.

11. Yoshikawa T, et al. (2021) Phase Gradients and Anisotropy of the Suprachiasmatic Network: Discovery of Phaseoids. eNeuro 8(5).

12. Belle MD, Diekman CO, Forger DB, \& Piggins HD (2009) Daily electrical silencing in the mammalian circadian clock. Science 326(5950):281-284.

13. Collins B, et al. (2020) Circadian VIPergic Neurons of the Suprachiasmatic Nuclei Sculpt the Sleep-Wake Cycle. Neuron 108(3):486-499 e485.

14. Meijer JH, Schaap J, Watanabe K, \& Albus H (1997) Multiunit activity recordings in the suprachiasmatic nuclei: in vivo versus in vitro models. Brain Res 753(2):322-327.

15. Colwell CS (2000) Circadian modulation of calcium levels in cells in the suprachiasmatic nucleus. Eur J Neurosci 12(2):571-576.

16. Ikeda $\mathrm{M}$, et al. (2003) Circadian dynamics of cytosolic and nuclear Ca2+ in single suprachiasmatic nucleus neurons. Neuron 38(2):253-263.

17. Noguchi T, et al. (2017) Calcium Circadian Rhythmicity in the Suprachiasmatic Nucleus: Cell Autonomy and Network Modulation. eNeuro 4(4).

18. Jones JR, Simon T, Lones L, \& Herzog ED (2018) SCN VIP Neurons Are Essential for Normal LightMediated Resetting of the Circadian System. J Neurosci 38(37):7986-7995.

19. Mei L, et al. (2018) Long-term in vivo recording of circadian rhythms in brains of freely moving mice. Proc Natl Acad Sci U S A 115(16):4276-4281.

20. Hong JH, et al. (2010) Intracellular calcium spikes in rat suprachiasmatic nucleus neurons induced by BAPTA-based calcium dyes. PLoS One 5(3):e9634.

21. Irwin RP \& Allen CN (2007) Calcium response to retinohypothalamic tract synaptic transmission in suprachiasmatic nucleus neurons. J Neurosci 27(43):11748-11757.

22. Hughes ME, Hogenesch JB, \& Kornacker K (2010) JTK_CYCLE: an efficient nonparametric algorithm for detecting rhythmic components in genome-scale data sets. J Biol Rhythms 25(5):372-380.

23. Carrillo-Reid L \& Yuste R (2020) Playing the piano with the cortex: role of neuronal ensembles and pattern completion in perception and behavior. Curr Opin Neurobiol 64:89-95.

24. Kaiser MI (2018) Individuating Part-Whole Relations in the Biological World. Individuation, Process, and Scientific Practices, eds Bueno O, Chen R-L, \& Bonnie Fagan M (Oxford Scholarship Online).

25. Ko CH, et al. (2010) Emergence of noise-induced oscillations in the central circadian pacemaker. PLoS Biol 8(10):e1000513.

26. Ikeda M \& Ikeda M (2014) Bmal1 is an essential regulator for circadian cytosolic $\mathrm{Ca}(2)(+)$ rhythms in suprachiasmatic nucleus neurons. J Neurosci 34(36):12029-12038.

27. Harvey JRM, Plante AE, \& Meredith AL (2020) Ion Channels Controlling Circadian Rhythms in Suprachiasmatic Nucleus Excitability. Physiol Rev 100(4):1415-1454.

28. Boulware MJ \& Marchant JS (2008) Timing in cellular Ca2+ signaling. Curr Biol 18(17):R769R776.

29. Gau D, et al. (2002) Phosphorylation of CREB Ser142 regulates light-induced phase shifts of the circadian clock. Neuron 34(2):245-253.

30. Deisseroth K \& Tsien RW (2002) Dynamic multiphosphorylation passwords for activitydependent gene expression. Neuron 34(2):179-182. 
31. Abrahamson EE \& Moore RY (2001) Suprachiasmatic nucleus in the mouse: retinal innervation, intrinsic organization and efferent projections. Brain Res 916(1-2):172-191.

32. Lee IT, et al. (2015) Neuromedin s-producing neurons act as essential pacemakers in the suprachiasmatic nucleus to couple clock neurons and dictate circadian rhythms. Neuron 85(5):1086-1102.

33. Morris EL, et al. (2021) Single-cell transcriptomics of suprachiasmatic nuclei reveal a Prokineticin-driven circadian network. EMBO J 40(20):e108614.

34. Wen S, et al. (2020) Spatiotemporal single-cell analysis of gene expression in the mouse suprachiasmatic nucleus. Nat Neurosci 23(3):456-467.

35. Xu P, et al. (2021) NPAS4 regulates the transcriptional response of the suprachiasmatic nucleus to light and circadian behavior. Neuron 109(20):3268-3282 e3266.

36. Gizowski C, Zaelzer C, \& Bourque CW (2016) Clock-driven vasopressin neurotransmission mediates anticipatory thirst prior to sleep. Nature 537(7622):685-688.

37. Jones JR, Chaturvedi S, Granados-Fuentes D, \& Herzog ED (2021) Circadian neurons in the paraventricular nucleus entrain and sustain daily rhythms in glucocorticoids. Nat Commun 12(1):5763.

38. Todd WD, et al. (2018) A hypothalamic circuit for the circadian control of aggression. Nat Neurosci 21(5):717-724.

39. Yulyaningsih E, et al. (2014) Pancreatic polypeptide controls energy homeostasis via Npy6r signaling in the suprachiasmatic nucleus in mice. Cell Metab 19(1):58-72.

40. Stowie A, et al. (2021) Arginine-Vasopressin Expressing Neurons in the Murine Suprachiasmatic Nucleus Exhibit a Circadian Rhythm in Network Coherence in vivo. BioRxiv https://doi.org/10.1101/2021.12.07.471437.

41. van der Horst GT, et al. (1999) Mammalian Cry1 and Cry2 are essential for maintenance of circadian rhythms. Nature 398(6728):627-630.

42. Bur IM, et al. (2009) The circadian clock components CRY1 and CRY2 are necessary to sustain sex dimorphism in mouse liver metabolism. J Biol Chem 284(14):9066-9073.

43. Miller JE, Ayzenshtat I, Carrillo-Reid L, \& Yuste R (2014) Visual stimuli recruit intrinsically generated cortical ensembles. Proc Natl Acad Sci U S A 111(38):E4053-4061. 


\section{Figure legends}

Fig. 1: Daily diversity and unity of two $\mathrm{Ca}^{2+}{ }_{i}$ dynamics in individual SCN neurons in vivo. a., Time-lapse projection of GCamp6f fluorescence monitored every two hours from zeitgeber time $Z T=0$ to $Z T=22$, through a GRIN lens aimed at the SCN of a freely-moving mouse. Scale bar, $100 \mu \mathrm{m}$. b, Daily variations in global GCamp6f signal intensity from one representative SCN field of view. The solid line represents the best cosine fit of experimental data points, measured every 30 minutes over four consecutive daynight cycles. c, Longitudinal one-minute recordings of GCamp6f fluorescence over one day-night cycle, from two SCN neurons within the same field of view. $\mathbf{d - i}$, Daily variation in spike frequency (d-f) and GCamp6f baseline (g-i), in SCN neurons. Example from one representative neuron $(\mathbf{d}, \mathbf{g})$, heat maps obtained from 52 neurons recorded in the same $\operatorname{SCN}$ field of view $(e, h)$, and Rayleigh plots of daily peak phases measured from four different mice $(\mathbf{f}, \mathbf{i})$. Each color represents neurons from one mouse. The inner circle represents the statistical threshold $(p<0.05)$ for the mean vector of the circular distribution of the aggregate data. The white and black boxes depict the light and dark phase, respectively. j, Phase-lag relationship between spike frequency and the GCamp6f baseline.

Fig. 2: Small $\mathrm{Ca}^{2+}{ }_{i}$ spikes built upon large daily changes in basal $\mathrm{Ca}^{2+}{ }_{i}$ levels. a., One-minute recording of GCamp6f fluorescence, with thresholds for the $5^{\text {th }}$ and $95^{\text {th }}$ percentiles (baseline and topline, black and red triangles, respectively) used to calculate the signal amplitude. $\mathbf{b}$-d, Daily variations in GCamp6f signal amplitude in the same neuron (b) and same SCN field (c) as in Fig. 1. d, Rayleigh plot of daily peak phases in signal amplitude for the aggregate data. e, Phase relationship between spike frequency and signal amplitude. f, Distribution of the ratio values calculated, for each one-minute recording, between the daily amplitude in baseline GCamp6f $\left(\Delta \mathrm{F}_{24 h}\right)$ and the signal amplitude over one minute $\left(\Delta \mathrm{F}_{1 \mathrm{~min}}\right)$, for each mouse SCN (colored lines) and the aggregate data (black bars). $\mathbf{g}, \mathbf{h}$, Daily profiles in GCamp6f baseline and topline (as depicted in a) from two representative SCN neurons, with higher signal amplitude during the day (g) or night (h). The solid lines represent the best cosine wave for each dataset.

Fig. 3: Synchronous fast $\mathrm{Ca}^{2+}{ }_{i}$ spikes depicted clock-independent cell-cell signaling in the SCN. a, Oneminute recordings of GCamp6f fluorescence from SCN neurons in a same field of view. Note the occurrence of synchronous spikes in neuron subsets (delineated by three different colors). Scale bar, $100 \mu \mathrm{m} . \mathbf{b}$, Global GCamp6f fluorescence (upper panel) and heat map of signal intensity from individual neurons (lower panel), recorded from a control mouse SCN, during one minute. c,d, Extent of clusters of co-activity as a function of the density in activity (c) and time of day (d). e, Global GCamp6f 
fluorescence (upper panel) and heat map of signal intensity from individual neurons (lower panel), recorded from a Cry1-/- Cry2-/- mouse SCN, during one minute.

Fig. 4: Schematic summary of the unity and diversity of $\mathrm{Ca}^{2+}{ }_{i}$ dynamics in the mouse $\mathrm{SCN}$ in vivo. Highly coordinated daily waves in baseline $\mathrm{Ca}^{2+}{ }_{i}$ levels (black sine lines) bear multiple patterns in fast $\mathrm{Ca}^{2+}{ }_{i}$ spikes (colored histograms), revealing neurons preferentially active during daytime (yellow and green) or nighttime (purple). Fast $\mathrm{Ca}^{2+}{ }_{\mathrm{i}}$ spikes also revealed extensive clock-independent intercellular signaling, involving circuit-level interactions between SCN cells and/or coordinated inputs from other brain regions. The combination of fast and slow $\mathrm{Ca}^{2+}{ }_{i}$ dynamics provides support for a multiplex neuronal code to pace different body rhythms. Created with BioRender.com 


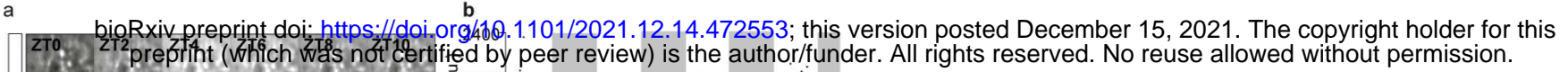
$\begin{array}{lllllll}\text { ZT12 } & \text { ZT14 } & \text { ZT16 } & \text { ZT18 } & \text { ZT20 } & \text { ZT22 }\end{array}$
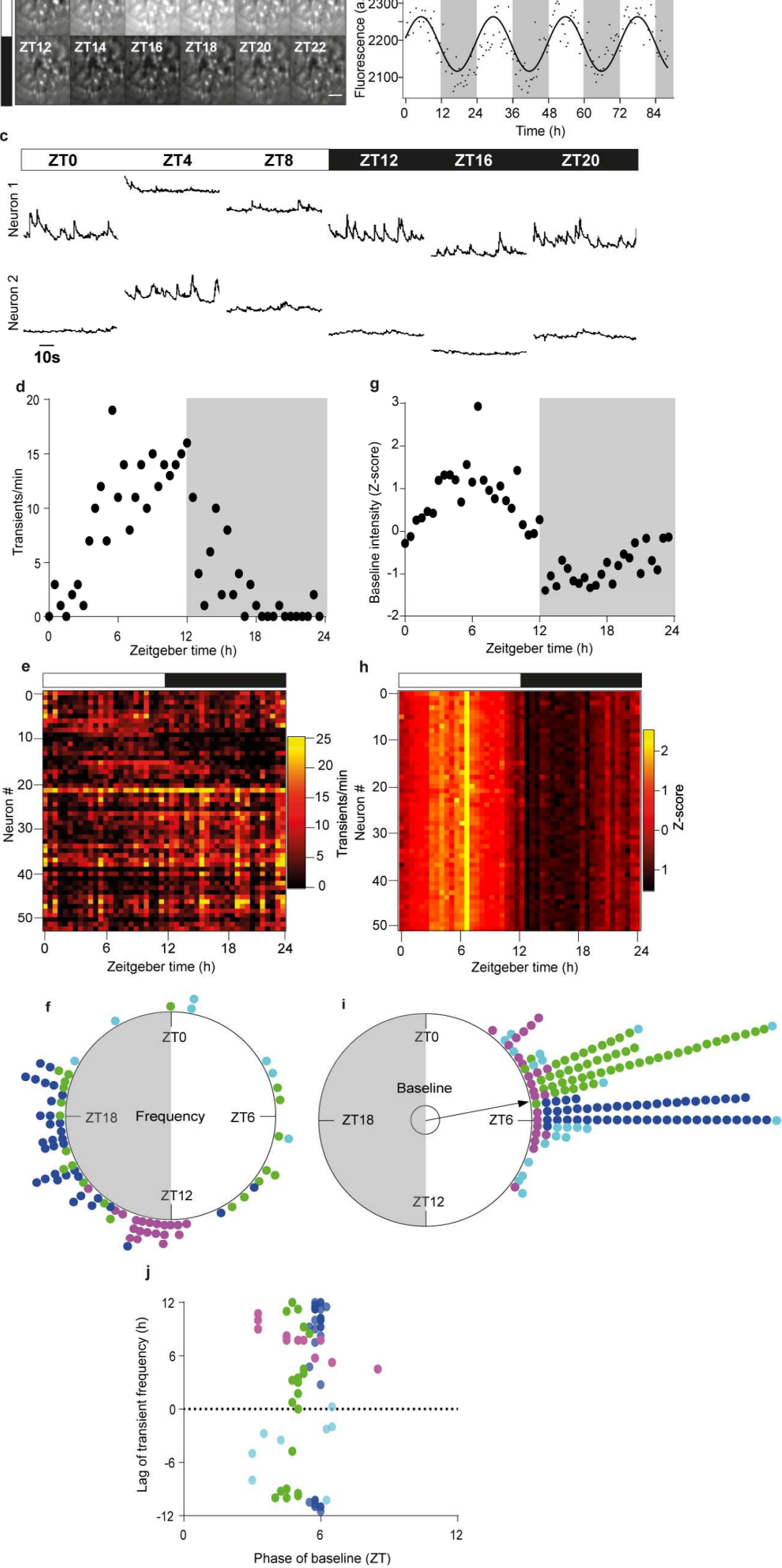
bioRxiv preprint doi: https://doi.org/240.1101/2021.12.14.472553; this version posted December 15, 2021. The copyright holder for this
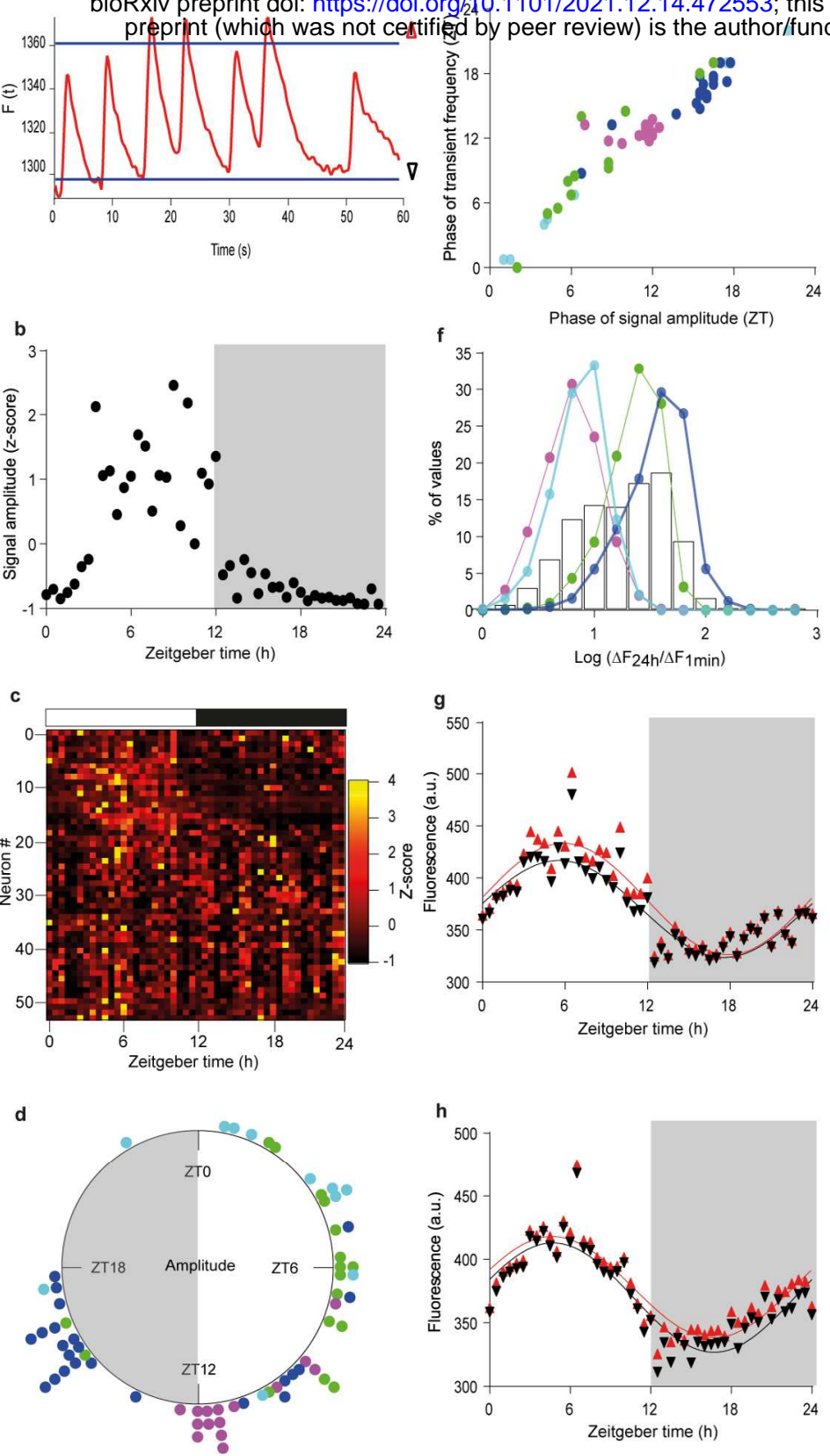
bipRxiv preprint do: https://doi.org/10.1101/2021.12.14.472553; this version posted December 15,2021 . The copyright holder for this
preprint (which was not certified by peer review) is the author/funder. All rights reserved. No reuse allowed without permission.
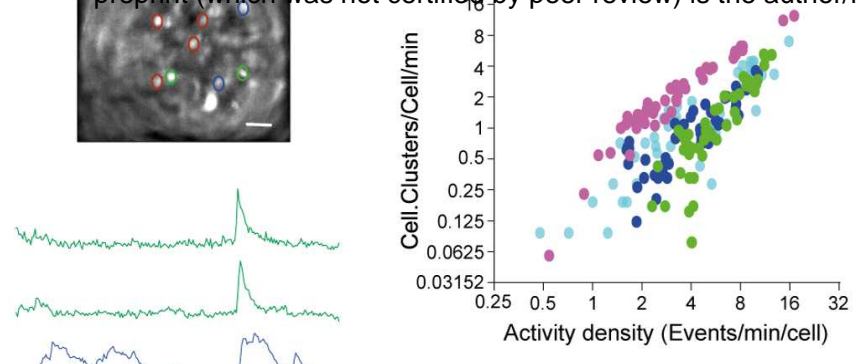

d Cell.Clusters/Cell/min
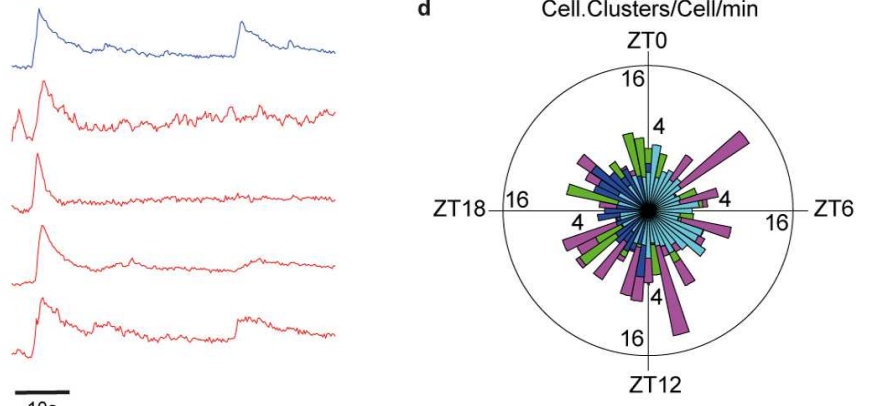

10s

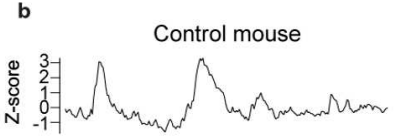

e Cry $1 /$ Cry2 deficient mouse
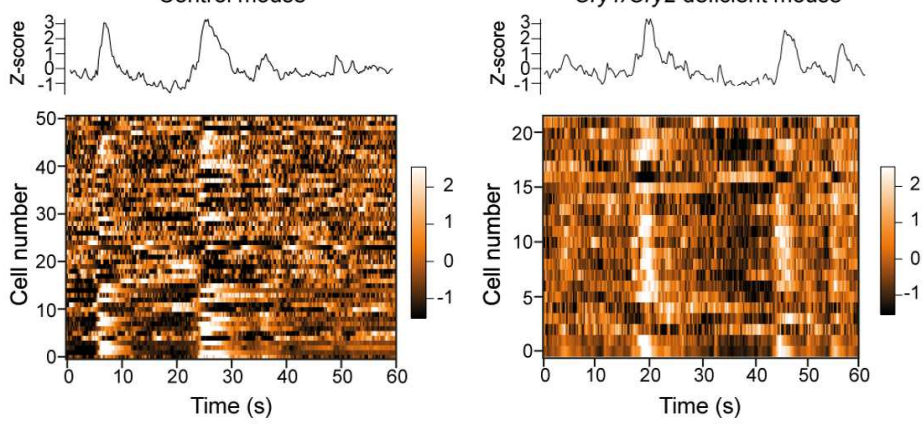

Figure 3 
bioRxiv preprint doi: https://doi.org/10.1101/2021.12.14.472553; this version posted December 15, 2021. The copyright hold/Ar for this

preprint (which was not certified by peer review) is the author/funder. All rights reserved. No reuse allowed without permission.

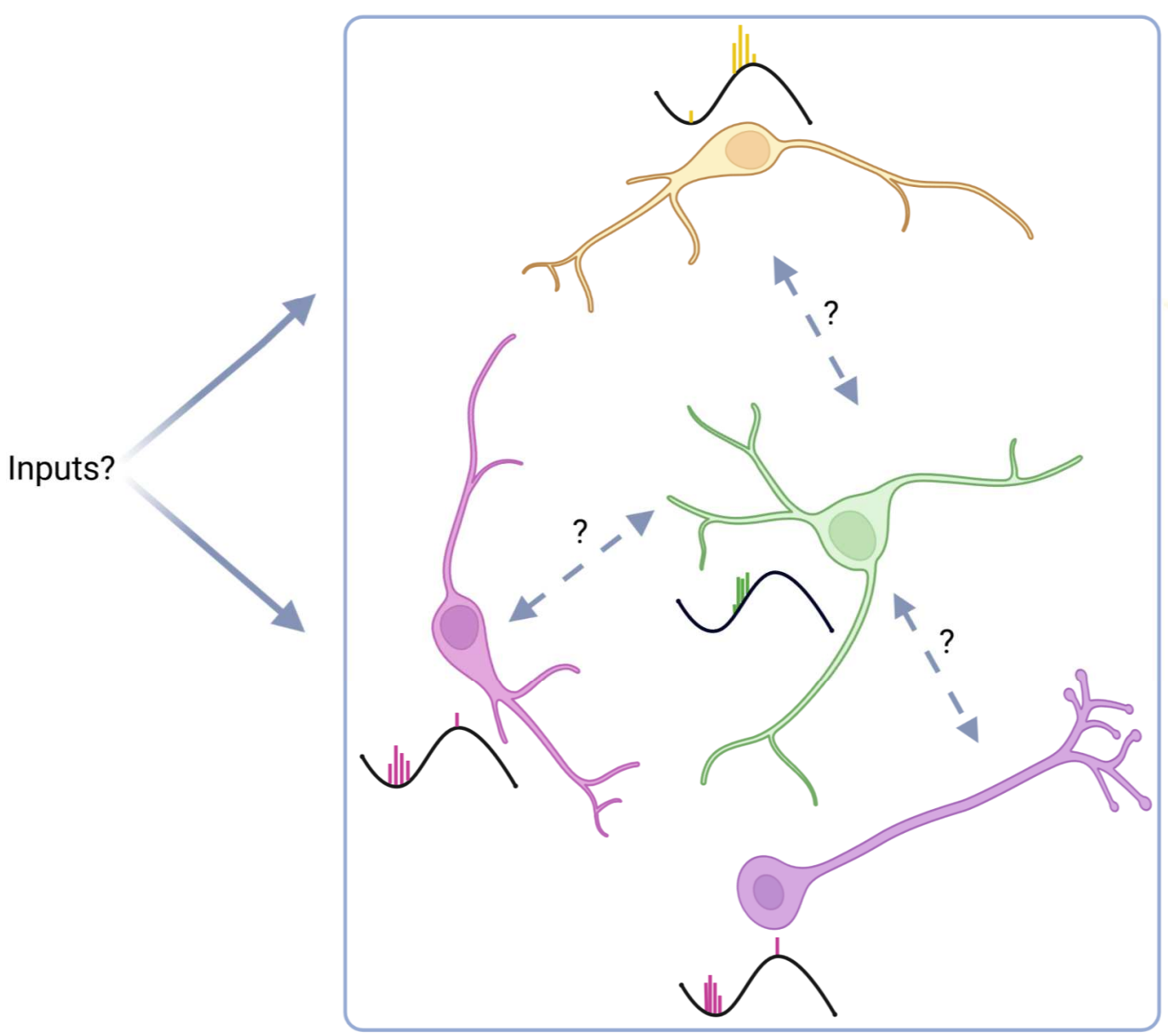

Different

functions? 